\title{
Visual demonstration of transmission electron microscopy for intracellular observation of a single bacterial cell
}

\author{
Shruti Shukla1 ${ }^{1}$ and Vivek K. Bajpai ${ }^{2}$ \\ ${ }^{1}$ Department of Food Science and Technology, Yeungnam University, Gyeongsan-si, Gyeongsanbuk-do, $712-$ \\ 749, Republic of Korea; ${ }^{2}$ Department of Applied Microbiology and Biotechnology, Yeungnam University, \\ Gyeongsan, Gyeongbuk 712-749, South Korea.
}

Correspondence to Vivek K. Bajpai at vbiotech04@gmail.com

First Published: 1 March, 2017

DOI: $10.3329 /$ bjp.v12i1.31390

\section{ABSTRACT}

Transmission electron microscopy (TEM) has a profound impact on knowledge and understanding of bacteria and other microbial populations. The 1000-fold improvement in resolution provided by TEM has allowed visualization of bacterial cells. Of all the types of microscopy exploited to date, TEM is the one with the most advantageous resolution limit and therefore it is a very efficient technique for deciphering the cell architecture and relating it to function. This visual experiment aims to provide an overview of the most important technique, TEM that we can apply to a biological sample, tissue or cells, to observe it with an internal structure, from the most conventional to the latest generation. Processes and concepts are defined, and the advantages and disadvantages of the TEM technique are assessed along with the image and information that we can obtain by using each one of them.

\section{INTRODUCTION}

The resolution of light and compound microscopes is limited to a maximum resolution of 200-400 nm, which only allows the observation of the general morphology of bacterial cells (Shukla, 2015), but not subcellular bacterial structures (Willey et al., 2008; Vale et al., 2010). A huge improvement in resolution is achieved with transmission electron microscopy (TEM) by replacing the large wavelength light beam with an electron beam that has a wavelength of approximately $0.005 \mathrm{~nm}$. Using TEM, electron beams are generated either by a tungsten filament, or by a field emission gun, substitute visible light which go through magnetic fields that work as lenses (Ruska, 1987; Agar et al., 1974). Electron beams are notoriously unable to penetrate through matter, and therefore only the thinnest possible specimens kept under high vacuum are suitable for imaging with this type of microscope. This posed enormous constraints to the development of the field and a complex array of technical achievements in producing very thin samples had to mature before relevant data could be extracted from electron microscopic images (Agar et al., 1974; Glauert, 1988). The images of biological samples produced by the electron microscope are inherently of very low contrast, since organic matter is made of relatively light atoms of carbon, oxygen and hydrogen that have low electron scattering power.

Staining methods requiring the introduction of heavy atoms into the specimen have to be employed in order to reveal ultrastructural detail (Hayat, 2000). Positive staining, shadow casting, and negative staining procedures were developed for visualization of thin sections and particulate samples. The maximum useful magnification a microscope is able to achieve is set by the resolution, as the goal is to make visible to the human eye the smallest object that the microscope can image (the size of the resolution limit). Since the human eye can distinguish objects down to $0.2 \mathrm{~mm}$, the useful magnification for a microscope with a resolution of $0.2 \mu \mathrm{m}$ (light microscope), is about $0.2 \mathrm{~mm} / 0.2 \mu \mathrm{m}$ or $1,000 \mathrm{x}$. The resolution of $0.2 \mathrm{~nm}$ of the TEM allows for a useful magnification of 1,000,000x, a factor 1000x higher than for the light microscope (Willey et al., 2008).

Therefore, the major objective of this visual experiment is to demonstrate the sample preparation, instrument handling and image visualization of bacteria by using step-by-step magnification process of transmission electron microscopic (TEM) analysis. 


\section{MATERIALS AND EQUIPMENTS}

Reagents and Solutions

Bacterial culture broth

Sterilized nutrient broth $100 \mathrm{~mL}$

Bacterial stock $\quad 100 \mu \mathrm{L}$

Phosphate buffer solution (PBS) 0.05M (pH 7.4)

Potassium phosphate monobasic (Sigma) $\quad 0.680 \mathrm{~g} / 100 \mathrm{~mL}$ distilled water

Potassium phosphate dibasic (Sigma) $\quad 0.871 \mathrm{~g} / 100 \mathrm{~mL}$ distilled water

Drop wise mix monobasic solution into dibasic solution until the $\mathrm{pH}$ reaches to 7.2.

Make up to $100 \mathrm{~mL}$ in a volumetric flask.

Oral acetate (2\%)

Oral acetate: $\quad 2.0 \mathrm{~mL}$

Triple Distilled water: $\quad 98.0 \mathrm{~mL}$

Disposables

Sterile plastic pipettes

Tips for micro-pipettes

Sterile Eppendorf tubes $(1.5 \mathrm{~mL})$

Bacterial culture (based on the desired study)

\section{General Equipment and Glassware}

Autoclave to sterilize the necessary materials

Laminar bench

Incubator to maintain optimum temperature for bacterial growth

Measuring cylinder $(100 \mathrm{~mL})$

Volumetric flask $(100 \mathrm{~mL})$

Sterilized forceps

Eppendorf tubes

Glass tubes (Size: $25.4 \times 76.2 \mathrm{~mm}$; Thickness: 1-1.2 mm)

Forceps sets

Metal coated grids

Major Equipment

Desktop centrifuge

Vortex

High power lamp based dryer

Transmission electron microscope (Hitachi S 4100) 


\section{VIDEO CLIPS}

Sample preparation: coating, staining and critical drying: $\quad 4$ min $36 \mathrm{sec}$

TEM instrumentation and image analysis: $\quad 4 \mathrm{~min} 37 \mathrm{sec}$

\section{METHOD}

\section{Preparation of bacterial cell culture}

1. Prepare sterilized nutrient broth in a conical glass flask $(250 \mathrm{~mL})$ for bacterial stock culture inoculation.

2. Inoculate $100 \mu \mathrm{L}$ of bacterial stock culture (approximately $10^{7} \mathrm{CFU} / \mathrm{mL}$ ) into the nutrient broth $(100 \mathrm{~mL})$ under laminar bench in a sterilized condition.

3. Incubate the inoculated broth in the incubator at $37^{\circ} \mathrm{C}$ for $18-20$ hours.

\section{Bacterial sample preparation of TEM}

1. Take out the bacterial culture from the incubator and place it into the laminar bench.

2. Transfer $900 \mu \mathrm{L}$ of bacterial culture in Eppendorf tubes for sample treatment as well as for control.

3. Centrifuge the cultures at $3000-4000 \mathrm{rpm}$ for $5 \mathrm{~min}$.

4. Discard the supernatant and wash the pellet using $1 \mathrm{~mL}$ of phosphate buffer solution (PBS) $0.05 \mathrm{M}$ (pH 7.4).

5. Repeat the process of washing with the same buffer and finally collect the pellet of both treatment and control separately.

[We can adjust the concentration of bacterial cell culture as per the image requirement as if we require a bunch of bacterial cells, use high concentration of pellet and if required single or separated bacterial cells, dilute the pellet in 0.05M PBS buffer ( $\mathrm{pH} 7.4$ ). In the present study, we used both high concentration of bacterial cells and diluted bacterial cells].

\section{Coating and staining of sample on metallic grid for morphological analysis}

1. About $10 \mu \mathrm{L}$ of the bacterial suspension to be studied is applied to the hydrophobic surface of a parafilm square in a Petri-dish

1. A formvar-coated grid is floated onto this drop for one min, with the formvar side of the grid in contact with the liquid.

2. The excess liquid is removed from the grid by touching its border with a cut piece of filter paper.

3 . The grid is immediately floated in a drop of $2 \%$ aqueous uranyl acetate, depending on the specimen.

4. After staining for one minute, the excess stain is removed with filter paper and the grid if left to dry for a few (10) minutes.

5. Then, remove and place the sample on another petri plate layer with filter paper for soaking of extra solution.

6. Keep the entire sample coated metallic grid for drying under the high power voltage lamp.

[For a better assessment of the samples, two or three grids should be prepared, each stained with one of these stains. Common mistakes, such as freezing the sample or leaving it unfixed for even "short-periods" of time can ruin the sample and are to be avoided at all cost.]

\section{Scanning electron microscopic instrumentation and analysis}

1. Perform drying of the coated sample on metallic grid.

2. After drying, fix the grid on to the tilt holder.

3. Place the holder attached to sample grid into the machine and start instrument following vacuum precautions and other instructions as showing in video. 
4. Place the coated slide on field emission -Scanning electron microscope.

5. Observe the image by setting photo speed, contrast/ brightness and image size.

[Use magnifications tools until search better image position and visuallity]

\section{DISCUSSION}

The preparation of a biological sample, cells or tissue, for transmission electron microscopy (TEM) requires several steps, some of which are quite complicated and some are critical. There are wide range of possible techniques and processes and the use of one particular process rather than another depending on the sample (type of tissue, cells, biofilm, etc.), size of the specimen being studied, how the sample is obtained (in a laboratory, a hospital, or in the field, etc.), the equipment that is available to us and finally, and very importantly, on what type of study we wish to carry out (ultrastructural, molecular localization, immunolocalization, electron tomography, correlative techniques, etc.) (Cortadellas et al., 2010). There are different possibilities for each type of study and before starting, it is necessary to decide which the best option is or options that we can use based on the information that we want to obtain from the sample and on the equipment that is available to us to carry out the study.

The first stage of preparing a biological sample for TEM is the fixation, one of the most important and most critical stages. The objective of this step is to halt cellular activity without altering the cellular characteristics, the components that make up the sample or their distribution; to preserve the internal three-dimensional organization, the size and the shape of the sample. The fixation process can be carried out in two different ways, by chemical fixation or by cryofixation (Mascorro and Bozzola, 2007). Several researchers have described a number of chemical methods for staining bacterial cells including the tannic acid-fuchsin method and modifications of this method (Kodaka et al., 1982; Grossart et al., 2000), as well as silver staining methods (Porter et al., 1992). These approaches are satisfactory but involve complicated protocols. Also, each method has limitations, such as unstable reagents and fixation induced alterations. In this research, we utilized chemical fixation using $2 \%$ solution of oral acetate. Although electron microscopes require specialized training, students can be assisted by the professionals and learn TEM techniques. Accessibility and use of TEM technique may provide students the opportunity to observe a nano-sized world in incredible depth and detail.

\section{CONCLUSION}

Transmission electron microscopy (TEM) is essential to understand how cells and tissues work. It allows us to visualize structures with high resolution and to relate their functions of the components as well as being able to detect and localize molecules in situ. However, all methods have advantages and disadvantages and their applications should be planned carefully.

\section{ADVANTAGES}

A TEM is an impressive instrument with a number of advantages such as:

- TEM offers the most powerful magnification, potentially over one million times or more.

- TEM has a wide-range of applications and can be utilized in a variety of different scientific, educational and industrial fields.

- TEM provides information on element and compound structure.

- Images are high-quality and detailed.

- TEM is able to yield information of surface features, shape, size and structure.

- TEM is easy to operate with proper training.

\section{DISADVANTAGES}

Some cons of electron microscopes include:

- TEM is large and very expensive.

- Laborious sample preparation. 
- Potential artifacts from sample preparation.

- Operation and analysis require special training.

- Samples are limited to those that are electron transparent, able to tolerate the vacuum chamber and small enough to fit in the chamber.

- TEM requires special housing and maintenance.

- Images are black and white.

- TEM is sensitive to vibration and electromagnetic fields and must be housed in an area that isolates them from possible exposure.

- TEM requires constant upkeep, including maintaining voltage, currents to the electromagnetic coils and cooling water.

\section{REFERENCES}

Agar AW, Alderson RH, Chescoe D, Glauert AM. Principles and practice of electron microscope operation. In: Practical methods in electron microscopy. Glauert AM (ed). London, Elsevier Science and Technology, 1974.

Cortadellas N, Garcia A, Fernández E. Transmission electron microscopy in cell biology: Sample preparation techniques and image information. Handbook of instrumental techniques from CCiTUB. TEM Cell Biol. 2010, pp 1-11.

Glauert AM, Lewis PR. Biological specimen preparation for transmission electron microscopy. New Jersey, Princeton University Press, 1988.

Grossart HP, Steward GF, Martinez J, Azam F. A simple, rapid method for demonstrating bacterial flagella. Appl Environ Microbiol. 2000; 66: 3632-36.

Hayat MA. Principles and techniques of electron microscopy: Biological applications. Cambridge, Cambridge University Press, 2000.

Kodaka H, Armfield AY, Lombard GL, Dowell VR. Practical procedure for demonstrating bacterial flagella. J Clin Microbiol. 1992; 16: 948-52.

Mascorro JA, Bozzola JJ. Processing biological tissues for ultrastructural study. In: Electron microscopy. Methods and protocols. Methods in Molecular Biology. John Kuo (ed). Human Press, 2007.

Porter JR, Thomulka KW, Smith RA. Demonstrating bacterial flagella. Am Biol Technol. 1992; 54: 108-11.

Ruska E. The development of the electron microscope and of electron microscopy. Biosci Rep. 1987; 7: 60729.

Shukla S. Studying antimicrobial-induced morphostructural damage of bacteria by Scanning Electron Microscope. Bangladesh J Pharmacol. 2015; 10: 870-74.

Vale FF, Correia, AC, Matos, B, Moura Nunes JF, Alves de Matos AP. Applications of transmission electron microscopy to virus detection and identification. Microscopy: Science, technology, applications and education. Méndez-Vilas A, Díaz J (eds). 2010, pp 128-36.

Willey J, Sherwood L, Woolverton C. Prescott, Harley and Klein's Microbiology. 7th ed. London, McGraw-Hill, 2008.

\section{PRECAUTION}

Incubation temperature and time should be checked for test bacterial species.

Avoid any possible contamination during bacterial growth and inoculation of treatment sample.

Careful precautions should be taken while setting the vacuum level in the instrument. 


\section{Your feedback about this paper}

1. Number of times you have read this paper 0

2. Number of times you have seen the video clip 0

3. Quality of paper

Click

4. Your comments

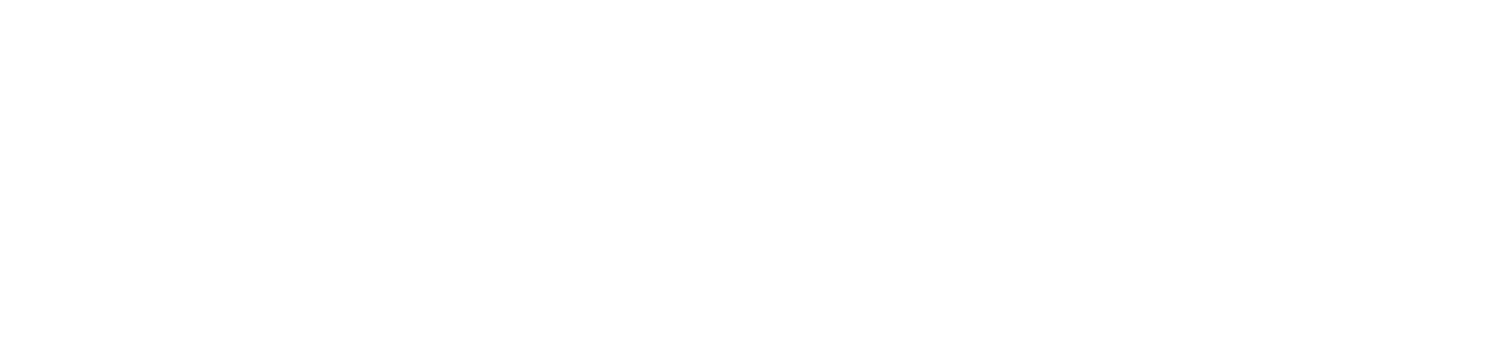

